\title{
Localization of Synucleins in the Mammalian Cochlea
}

\author{
O. Akil, ${ }^{1}$ C. M. Weber, ${ }^{1}$ S. N. Park, ${ }^{1,2}$ N. Ninkina, ${ }^{3}$ V. Buchman, ${ }^{3}$ and L. R. Lustig ${ }^{1}$ \\ ${ }^{1}$ Department of Otolaryngology—Head and Neck Surgery, University of California San Francisco, UC Hall, Room U401, \\ 533 Parnassus Avenue, San Francisco, CA 94143-0449, USA \\ ${ }^{2}$ Department of Otolaryngology—Head and Neck Surgery, The Catholic University of Korea, College of Medicine, Seoul, \\ South Korea \\ ${ }^{3}$ School of Biosciences, Cardiff University, Museum Avenue, Cardiff, CF10 3US, UK
}

Received: 21 March 2008; Accepted: 11 July 2008; Online publication: 30 July 2008

\begin{abstract}
Synucleins are widely expressed synaptic proteins within the central nervous system that have been implicated in such neurodegenerative disorders as Parkinson's disease. In this study, an initial characterization of all three synucleins, $\alpha-, \beta-$, and $\gamma$-synuclein, within the cochlea was undertaken. Reverse transcriptase-polymerase chain reaction (PCR) demonstrated all three synuclein mRNA species within microdissected cochlear tissue. Quantitative PCR suggests that $\beta$-synuclein is the most abundantly expressed form, followed by $\gamma$ - and then $\alpha$ synuclein. Western blot analysis similarly demonstrates all three synuclein proteins within microdissected cochlear tissue. Immunofluorescence localizes the three synucleins predominantly to the efferent neuronal system at the efferent outer hair cell synapse, with some additional localization within the efferent tunnel-crossing fibers ( $\alpha$ - and $\gamma$-synuclein), spiral ganglion ( $\beta$-synuclein), inner spiral bundle ( $\gamma$-synuclein), and stria vascularis $(\alpha->$ $\beta$-synuclein). Developmentally, $\gamma$-synuclein can be seen in the region of the outer hair cells by E19, while $\alpha$-and $\beta$ synuclein do not clearly appear there until $\sim$ P10. Additional studies in a null-mutant $\gamma$-synuclein mouse show no histological changes in the organ of Corti with normal hair cell and spiral ganglion cell counts, and normal ABR and DPOAE thresholds in wild-type vs mutant littermates. Together, these results localize synucleins to the efferent cholinergic neuronal auditory system, pointing to a role in normal auditory function, and raising the potential
\end{abstract}

Correspondence to: L. R. Lustig - Department of OtolaryngologyHead and Neck Surgery - University of California San Francisco · UC Hall, Room U401, 533 Parnassus Avenue, San Francisco, CA 941430449, USA. Telephone: +415-476-0728; fax: +415-476-0708; email: llustig@ohns.ucsf.edu implications for their role in auditory neurodegenerative disorders. However, $\gamma$-synuclein alone is not required for the development and maintenance of normal hearing through P21. Whether overlapping roles of the other synucleins help compensate for the loss of $\gamma$-synuclein remains to be determined.

Keywords: synuclein, alpha-synuclein, beta-synuclein, gamma-synuclein, $\alpha$-synuclein, $\beta$-synuclein, $\gamma$-synuclein, efferent, auditory, cochlea, nicotinic acetylcholine receptor, outer hair cell, inner hair cell, hearing, synapse

\section{INTRODUCTION}

The synuclein family includes three small, soluble, highly conserved neuronal proteins termed $\alpha-, \beta-$, and $\gamma$-synuclein (George 2002). These synaptic proteins have only been identified within vertebrates, with no known homologous proteins in non-vertebrate species. Despite the abundance of synucleins in the central nervous system (CNS), the precise role for these proteins remains uncertain. $\alpha$-Synuclein, in particular, has generated significant interest due to its presumed role in Parkinson's disease, multiple system atrophy, and other neurodegenerative "synucleinopathies" (Kahle 2007; Stichel et al. 2007). Both $\alpha$ - and $\beta$-synuclein have been shown to be highly specific inhibitors of phospholipase D2, which in turn produces phosphatidic acid, a plasma membrane and submembranous vesicle component (Jenco et al. 1998; Tofaris and Spillantini 2007). This had led to the hypothesis that synucleins may be involved in synaptic membrane biogenesis and vesicle budding (Tofaris and Spillantini 2007). Other recent 
studies have implicated $\alpha$-synuclein in glutamate release (Gureviciene et al. 2007). Additional work has demonstrated that $\alpha$-synuclein may be required for the genesis or maintenance of presynaptic neurotransmitter vesicle numbers and mobilization (Cabin et al. 2002; Murphy et al. 2000). The known functional roles for $\beta$ - and $\gamma$-synuclein are even less precise.

All three synucleins have been identified in human, rodent, bird, and bovine tissues (George 2002). Of the three synucleins, within rodent brain $\alpha$-synuclein is the mostly highly abundant species, followed by $\beta$-synuclein and then $\gamma$-synuclein. $\alpha$-Synuclein is expressed throughout the entire CNS and is most abundant in catecholaminergic regions. In contrast, $\beta$-synuclein is more localized in the somatic cholinergic components of the CNS, being weak or absent in the catecholaminergic neurons. $\gamma$-Synuclein is present in both cholinergic and catecholaminergic regions ( $\mathrm{Li}$ et al. 2002).

To date, synucleins have not been described within the peripheral auditory system. Yet, with the welldescribed role of cholinergic and catecholaminergic neuronal signaling within the organ of Corti (Eybalin 1993; Puel 1995), as well as synuclein's presumed role in $\mathrm{Ca}^{2+}$ homeostasis and effects on voltage-dependent $\mathrm{Ca}^{2+}$ influx (Adamczyk and Strosznajder 2006; Ueda et al. 1997), one might expect to see evidence of synucleins in the auditory system as well. This current work documents the presence of synucleins within the cochlea and explores the localization of the three members of the synuclein family within the organ of Corti. Lastly, physiologic studies on a null-mutant $\gamma$-synuclein mouse are presented to make some inferences with regard to the role of synucleins within the auditory system.

\section{METHODS}

\section{Animals}

The C57BL/ 6 wild mice and Sprague-Dawley rat were purchased from Charles River. $\gamma$-Synuclein null-mutant mice have been previously described (Ninkina et al. 2003). $\alpha$-Synuclein knockout mice were obtained from the Jackson Labs (http://jaxmice.jax.org, Stock\#003692, Bar Harbor, ME, USA) and crossed multiple times onto C57BL/6 for strain maintenance. All procedures and animal handling were done according to national ethic guidelines, approved and complied with all protocol requirements at the University of California, San Francisco (IACUC).

\section{Auditory brainstem response and otoacoustic emission testing}

Auditory testing including auditory brainstem response (ABR) and distortion product otoacoustic emissions (DPOAEs) were performed as previously described
(Akil et al. 2006) with P21 $\gamma$-synuclein knockout (KO) mice and wild-type (WT) littermates. Briefly, all auditory testing was performed in a sound-proof chamber. Before acoustic testing, mice were anesthetized by intraperitoneal injection of a mixture of ketamine hydrochloride (Ketaset, $100 \mathrm{mg} / \mathrm{kg}$ ) and xylazine hydrochloride (xyla-ject, $10 \mathrm{mg} / \mathrm{kg}$ ) and boosted with one fifth the original dose as required. Body temperature was maintained with a heating pad and monitored with a rectal probe throughout recording.

\section{ABR testing}

Evoked acoustic brainstem response thresholds were differentially recorded from the scalp of the mice at age P21. Responses were recorded using subdermal needle electrodes at the vertex, below the pinna of the left ear (reference), and below the contralateral ear (ground). The sound stimuli used included click ( 5 ms duration; $31 \mathrm{kHz})$ and tone pips at 8,16 , and $32 \mathrm{kHz}(10 \mathrm{~ms}$ duration; cos2 shaping; $21 \mathrm{kHz}$ ). Measurements were done using the TDT BioSig III system (Tucker Davis Technologies, Alachua, FL, USA). For each stimulus, electroencephalographic (EEG) activity was recorded for $20 \mathrm{~ms}$ at a sampling rate of $25 \mathrm{kHz}$, filtered $(0.3-$ $3 \mathrm{kHz}$ ), and waveforms from 512 stimuli were averaged for click responses, and 1,000 stimuli for frequencyspecific stimuli $(8,16$, and $32 \mathrm{kHz})$. ABR waveforms were recorded in $5 \mathrm{~dB}$ sound pressure level (SPL) intervals down from the maximum amplitude. Threshold by visual inspection was defined as the lowest stimulus level at which response peaks for wave $I-V$ were clearly present. Computerized analysis of stored waveforms for ABR threshold (Matlab software, Mathworks, Natick, MA, USA) was also performed. The mean value of thresholds checked by visual inspection and computer analysis was defined as ABR threshold at each stimulus. Data was obtained from wild-type $(n=6)$ and homozygous $\gamma$-synuclein nullmutant $(n=10)$ mice.

Comparison of mean values of ABR thresholds between wild-type and homozygous $\gamma$-synuclein nullmutant mice was performed with analysis of variance (ANOVA) using a Bonferroni analysis, and significance was defined as a $p$ value less than 0.05 .

\section{Distortion product otoacoustic emission testing}

The distortion product otoacoustic emissions (DPOAEs) were measured using an acoustic probe placed in the left external auditory canal. Stimuli consisted of two primary tones $\left(f_{1} / f_{2}=1.25\right)$ digitally synthesized at $100 \mathrm{kHz}$ using SigGen software (Tucker Davis Technologies). The primary tones with geometric mean (GM) frequencies ranging from 6 to $36 \mathrm{kHz}$ and equal levels $\left(L_{1}=L_{2}=\right.$ 60dBSPL) were presented via two separate speakers (EC1) to the acoustic probe. DPOAE $2 f_{1}-f_{2}$ responses 
were recorded using an ER10B + (Etymotics Research) microphone assembly within the acoustic probe and the TDT BioSig III system. Responses were amplified, digitally sampled at $100 \mathrm{kHz}$ and averaged over 50 discrete spectra. Fast-Fourier transforms were computed from averaged responses. For each stimulus set, DPOAE amplitude level at $2 f_{1}-f_{2}$ was extracted, and sound pressure levels for data points $100 \mathrm{~Hz}$ above and below the DPOAE frequency were averaged for the noise floor measurements (NF). DPOAE level was plotted as a function of primary tone GM frequency. Data was collected from the same set of mice as described above for ABR recordings. Statistical analysis for comparison of mean DPOAE levels at each GM frequency between two groups was done using Student $t$ test with significance defined as $p<0.05$.

\section{Reverse transcriptase-polymerase chain reaction}

The total RNA harvested from the mice cochlea extractions (Trizol ${ }^{\mathrm{TM}}$, Invitrogen Corp) was reverse transcribed with superscript II RNase $\mathrm{H}^{-}$(Invitrogen) for $50 \mathrm{~min}$ at $42^{\circ} \mathrm{C}$, using oligodT primers. Two microliters of RT reaction product was used for subsequent polymerase chain reaction (PCR; Taq DNA Polymerase, Invitrogen) of 35 cycles using the following parameters: $94^{\circ} \mathrm{C}$ for $30 \mathrm{~s}, 60^{\circ} \mathrm{C}$ for $45 \mathrm{~s}, 72^{\circ} \mathrm{C}$ for $1 \mathrm{~min}$, followed by a final extension of $72^{\circ} \mathrm{C}$ for $10 \mathrm{~min}$ and storage at $4^{\circ} \mathrm{C}$. Primers were designed to amplify a unique sequence of rat or mouse synucleins, each spanning intron-exon boundaries to ensure no genomic DNA was amplified. The PCR primers that were used for rat: $\alpha$ synuclein (Genbank accession number NM_019169) forward-gggaaaacaggaggaatcagag and reverseaggattccctcttgtgggtac were designed to amplify a 408-bp fragment spanning the 3 ' portion of exon 2 through the $5^{\prime}$ portion of exon 5, $\beta$-synuclein (NM 080777$)$ forwardgcgcgtctgctggcagtagccgc and reverse-cccttcaggctccatca gag primers were designed to amplify a 427-bp fragment spanning the $3^{\prime}$ portion of exon 1 through the $5^{\prime}$ portion of exon 4, $\gamma$-synuclein (NM_031688) forward - cccgtcca gttcgtagcttgca and reverse- -tctttggcctcctggtcctg primers were designed to amplify a 382-bp fragment spanning the $3^{\prime}$ portion of exon 1 through the $5^{\prime}$ portion of exon 4 . The PCR primers that were used for mice: $\alpha$-synuclein (NM_009221) forward - gtggagcaaaaatacatctttag and reverse - tgtacgccatggaagagcagc primers were designed to amplify a 505-bp fragment spanning exon 2 through the $5^{\prime}$ portion of exon 6 , $\beta$-synuclein (NM 033610 ) forwardctctggggctgggaacatt and reverse-acgctctggctcg tattcctggta primers were designed to amplify a 189-bp fragment spanning the $3^{\prime}$ portion of exon 3 through the $5^{\prime}$ portion of exon 5, $\gamma$-synuclein (BC028508) forwardccaggtccatccttgcaaaca and reverse-taggcagggcatt cagtgtgc primers were designed to amplify a 481-bp fragment spanning the 3 ' portion of exon 3 through the $5^{\prime}$ portion of exon 5. Controls included cochlea RNA without reverse transcriptase.

Analysis of each PCR sample was then performed on $2 \%$ agarose gels containing $0.5 \mu \mathrm{g} / \mathrm{ml}$ ethidium bromide. Gels were visualized using a digital Camera and image processing system (Kodak, Rochester NY, USA). Candidate bands were cut out and the DNA was extracted (Qiaquick gel extraction kit, Qiagen) and sequenced (Elim Biopharmaceuticals, Inc. Hayward, CA, USA). The PCR product was then compared directly to the full rat or mouse synuclein sequence for identity.

Quantitative PCR. For real-time, quantitative PCR, cochlea from wild C57BL/6 mice were dissected. RNA was extracted and processed as described above. The expression level of synucleins $(\alpha, \beta$, and $\gamma)$ was measured by the iQ5 real-time PCR (Bio-Rad, Hercules, CA, USA). Amplification was performed in a total volume of $25 \mu \mathrm{l}$ containing $12.5 \mu \mathrm{l}$ SYBR Green I amplification master mix (Bio-Rad), $250 \mathrm{nM}$ of each primer $\alpha$-synuclein (NM_009221) forward-gtggagcaaaaatacatctttag and reverse-tgggtacccctcctcaccctt primers were designed to amplify a 348-bp fragment spanning exon 2 through the $5^{\prime}$ portion of exon $5, \beta$ synuclein (NM_033610) forward-cccggcccgaaacc caggccg and reverse-cttcaggtctgtggggaactctt primers were designed to amplify a 305-bp fragment spanning the $3^{\prime}$ portion of exon 1 through exon $3, \gamma$-synuclein (BC028508) forward-ccaggtccatccttgcaaaca and reverse-ctttggcttcttggttcctgtg primers were designed to amplify a 354-bp fragment spanning the $3^{\prime}$ portion of exon 1 through the $5^{\prime}$ portion of exon 4 and ribosomal protein L19 (BC083131) forward-atgtatcacagcctgtacctg, and reverse - ttcttggtctcttcctccttg primers were designed to amplify a 232-bp fragment, and $3 \mu \mathrm{l}$ of 1:5 diluted cDNA as template. The amplifications were performed in duplicate wells, using a program that consisted of initial denaturation at $95^{\circ} \mathrm{C}$ for $3 \mathrm{~min}$ followed by a 40-cycle of denaturation at $95^{\circ} \mathrm{C}$ for $10 \mathrm{~s}$ and annealing at $61^{\circ} \mathrm{C}$ for $30 \mathrm{~s}$. After amplification, a melting curve was generated for every PCR product to check the specificity of the PCR reaction (absence of primer dimers or other nonspecific amplification product). The $2^{-\Delta \Delta C_{\mathrm{T}}}$ method was used to calculate relative fold difference in synuclein expression determined from real-time quantitative PCR experiments normalized to the ribosomal protein L19, an internal control, relative to the expression level of alpha synuclein. (Livak and Schmittgen 2001) Statistical differences were calculated using a one-way ANOVA with Bonfferoni post hoc test with significance noted for $p<0.05$.

\section{Mouse synuclein immunofluorescence}

C57BL6 wild mice at varying time points (E16, E19, P0, P2, P5, P10, P15, and P21) or P21 Sprague-Dawley rats 
were anesthetized, and their cochleae were isolated, dissected, perfused through the oval and round windows by $2 \%$ paraformaldehyde in $0.1 \mathrm{M}$ phosphate buffer (PB) at $\mathrm{pH} 7.4$ and incubated in the same fixative for $2 \mathrm{~h}$. After fixation, cochlease was rinsed with $\mathrm{PB}$ and immersed in $5 \%$ ethylenediaminetetraacetic acid (EDTA) in $0.1 \mathrm{M} \mathrm{PB}$ for decalcification. When cochleae were totally decalcified ( $\sim 2$ days), they were incubated overnight in $30 \%$ sucrose for cryoprotection. The cochleae then was embedded in O.C.T. Tissue Tek Compound (Miles Scientific). Tissues were cryosectioned at 10-12 $\mu \mathrm{m}$ thickness for immunofluorescence, mounted on Superfrost $^{\mathrm{TM}}$ microscope slides (Erie Scientific, Portsmouth $\mathrm{NH}$ ) and stored at $-20^{\circ} \mathrm{C}$ until used.

For immunofluorescence staining of the mice or rat cochlea, a rabbit polyclonal gamma synuclein antibody was made against the C-terminal end of mouse gamma synuclein (Buchman et al. 1998), a monoclonal anti- $\beta$ synuclein, clone Syn207 antibody, was made toward the full length recombinant human $\beta$-synuclein (Chemicon cat no. 36-009), and a monoclonal anti $\alpha$ synculein antibody was made against rat synuclein-1 (BD Biosciences cat no. 610786). After incubation at $37^{\circ} \mathrm{C}$ for $30 \mathrm{~min}$, the sections were rinsed twice for $5 \mathrm{~min}$ in $0.1 \mathrm{M}$ PBS pH 7.4, then preincubated for $1 \mathrm{~h}$ in $0.3 \%$ Triton X100 and 30\% normal goat serum (NGS). The sections were then incubated in a humid chamber overnight at $4^{\circ} \mathrm{C}$ with each anti-synuclein antibody with different concentrations as 1:300 $\alpha$-synuclein, 1:200 $\beta$-synuclein, and 1:150 $\gamma$-synuclein diluted in the blocking buffer. Slides were then rinsed $(2 \times 10 \mathrm{~min})$ and incubated for $2 \mathrm{~h}$ in goat anti-rabbit/mouse IgG conjugated to $\mathrm{Cy} 2$ or Cy3 diluted to $1: 4,000$. The sections were rinsed in PBS $(2 \times 10 \mathrm{~min})$ and mounted in equal parts glycerol/PBS and cover-slipped. Slides treated with the same technique but without incubation in the primary antibody were used as a control.

For double-labeling along with synucleins, tissue sections were prepared as described above. Additional markers and antibodies for staining were used as follows: choline acetyl transferase (ChAT, Chemicon)goat anti-choline acetyltansferase affinity purified polyclonal antibody was used at 1:75 and incubated overnight at $4^{\circ} \mathrm{C}$. In all experiments, the fluorescent dye 4,6-diamidino-2-phenylindole (DAPI, $1.5 \mu \mathrm{g} / \mathrm{ml}$ in PBS, Sigma) was also used to counterstain nuclei. Following the removal of the secondary antibodies and prior to the final washes, the sections were exposed for $15 \mathrm{~min}$ at room temperature to DAPI staining, which allowed visualization of the nuclei together with immunofluorescence. Neurofilament-200 (NF-200; Chemicon, Temecula, CA, USA) labels afferent as well as some efferent auditory fibers (Berglund and Ryugo 1991), and synaptophysin (Zymed, South San Francisco, CA, USA), predominantly labels efferent auditory fibers (Schimmang et al. 2003). After incubation at $37^{\circ} \mathrm{C}$ for
$30 \mathrm{~min}$, the sections were rinsed twice for $5 \mathrm{~min}$ in $0.1 \mathrm{M}$ PBS pH 7.4, then preincubated for $1 \mathrm{~h}$ in either $0.3 \%$ Triton X100 and 30\% normal goat serum (NGS; NF200), or $10 \%$ NGS without Triton X100 (synaptophysin). The sections were then incubated in a humid chamber overnight at $4^{\circ} \mathrm{C}$ with the primary antibody (1:500 NF200 or 1:200 synaptophysin diluted in PBS). They were then rinsed $(2 \times 10 \mathrm{~min})$ and incubated for $2 \mathrm{~h}$ in goat anti-rabbit IgG conjugated to $\mathrm{Cy} 2$ or $\mathrm{Cy} 3$ diluted to 1:4,000 in PBS. The sections were rinsed in PBS $(2 \times$ $10 \mathrm{~min}$ ) and mounted in equal parts glycerol/PBS and cover-slipped. Slides treated with the same technique but without incubation in the primary antibody were used as a control. Slides were observed with an Olympus microscope with confocal immunofluorescence.

\section{Western blot analysis}

Total protein extract was prepared from rat and mice cochlea using a lysis buffer containing $(20 \mathrm{mM}$ Tris $\mathrm{HCl}$ pH 7.5-8, 150 mM NaCl, 0.5\% sodium deoxycolate, $1 \%$ Triton X100, 0.1 sodium dodecyl sulfate (SDS), $1 \mathrm{mM}$ EDTA, $1 \mathrm{mM}$ phenylmethylsulphonyl fluoride, and protease inhibitor cocktail). The cochlea homogenate was spun $10 \mathrm{~min}$ at $14,000 \mathrm{RPM}$ at $4^{\circ} \mathrm{C}$. The protein concentration in the supernatant was determined by Bradford assay. Approximately $100 \mu \mathrm{g}$ of the supernatant was fractioned by SDS-polyacrylamide gel electrophoresis (PAGE) and transferred onto nitrocellulose. The blots were incubated for $1 \mathrm{~h}$ in blocking buffer containing 5\% nonfat dry milk in PBS-T (phosphate buffer saline $+0.1 \%$ Tween 20) and then incubated overnight at $4^{\circ} \mathrm{C}$ with various synuclein antibodies used for immunohistochemistry diluted in 1\% BSA in PBS-T ( $\alpha$ - and $\gamma$-synucleins 1:500, $\beta$-synuclein 1:5,000). The blots were washed in PBS-T and incubated for $2 \mathrm{~h}$ at room temperature with the secondary antibody (antimouse IgG peroxidase conjugate Sigma cat no. A4416) 1:5,000 for $\alpha$ - and $\beta$-synucleins and anti-rabbit IgG peroxidase conjugate GE (Healthcare cat no. NA934, 1:3,000). The blots were washed again with PBS-T, dried, and then incubated for $1 \mathrm{~min}$ with the Chemiluminescence reagent NEL 103 (Perkin Elmer). After 1 min, the membranes were dried and exposed to KODAK XOMAT blue film. Then, the films were developed for protein visualization.

\section{RESULTS}

Localization of synucleins within the organ of Corti

Reverse-transcriptase (RT)-PCR was initially used to screen for the presence of the three synucleins within the cochlea. As shown (Fig. 1), all three synuclein mRNA species were identified in both rat and mouse 


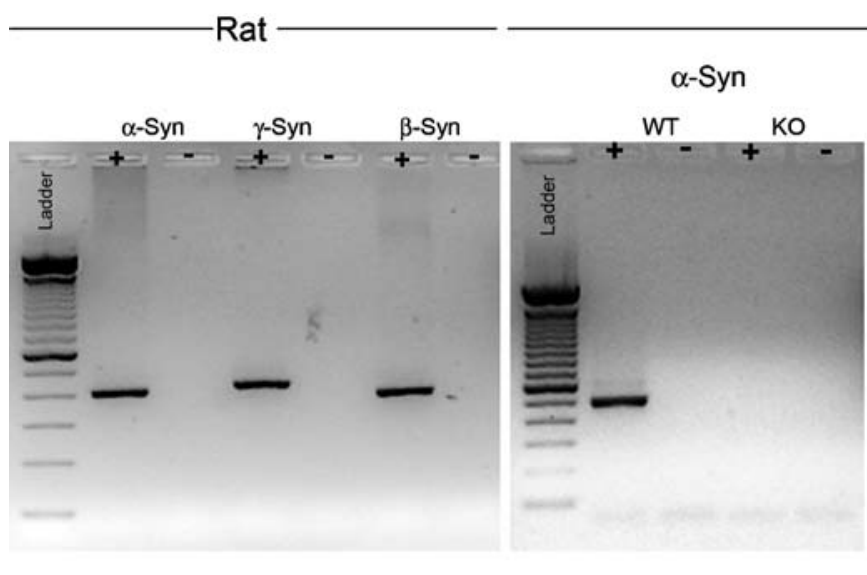

FIG. 1. RT-PCR of synucleins within the Cochlea. RT-PCR was used to detect the presence of $\alpha-, \beta-$, and $\gamma$-synuclein mRNA in microdissected rodent (rat-left panel, mouse-three central panels) cochlear tissue. Controls lacking reverse-transcriptase (denoted with $\left.\mathrm{a}^{\prime}-^{\prime}\right)$ are included. Both an $\alpha$ - and $\gamma$-synuclein knockout (KO) mouse

whole cochlear extracts. Simultaneous studies on microdissected organ of Corti from both an acquired $\alpha$ synuclein knockout and $\gamma$-synuclein knockout mouse appropriately demonstrate that neither of these mice produce their respective null-mutation synuclein mRNA. Quantitative PCR (qPCR) was next used to assess the relative changes of the three synucleins; seven separate experiments from different animals using two cochlea per study were analyzed in this manner. These studies suggest that $\beta$-synuclein is the most abundant species, followed by $\gamma$-synuclein and then $\alpha$-synuclein. Differences between the expression levels of each synuclein were statistically significant $(p<0.05)$.

To demonstrate the presence of translated synuclein proteins within similar microdissected cochlear tissue, Western blot analysis was performed. As shown (Fig. 2), all three synuclein proteins are found within the cochlea. Again, similar studies in the $\alpha$ - and $\gamma$-synuclein nullmutant mice (Fig. 2, lanes 4 and 5, respectively) demonstrate both the specificity of the antibodies used as well as the correct null phenotype of the knockout mice.

Immunofluorescence was next used to localize the three synucleins within the cochlea (Fig. 3). $\alpha$-Synuclein localized predominantly to the synaptic base of the outer hair cells and the efferent tunnel-crossing fibers, and more weakly to the base of the inner hair cell, as well as within the stria vascularis (Fig. $3 \mathrm{a}, \mathrm{d}, \mathrm{g}, \mathrm{h}, \mathrm{k}$ ). In contrast, $\beta$-synuclein most prominently localized to the base of the outer hair cells and spiral ganglion, and more weakly within the stria vascularis (Fig. 3B, E, I, L). $\gamma$-Synuclein had the most restrictive localization; antibodies against $\gamma$-synuclein most strongly label the base of the outer hair cells with some labeling of the efferent tunnel-crossing fibers, as well as the inner spiral bundle (Fig. 3c). $\gamma$-synuclein co-localized with $\alpha$ synuclein at the synaptic base of the outer hair cell
Mouse
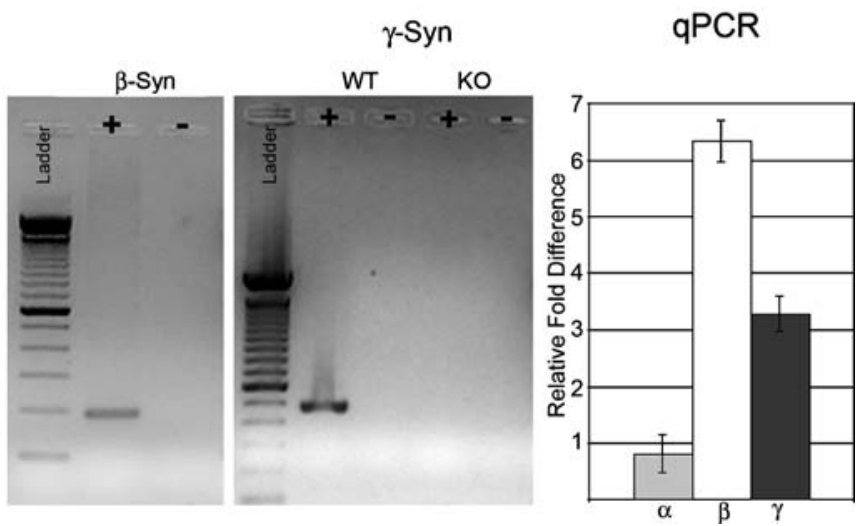

were also studied, demonstrating the correct phenotype of the nullmutant mouse. Quantitative PCR ( $q P C R$ - right panel) was used to assess relative mRNA differences of the three synucleins $(\alpha, \beta, \gamma)$ within microdissected cochlear tissue. WT Wild-type, $K O$ knockout mice.

(Fig. 3g, yellow staining), but showed no staining within the stria vascularis nor spiral ganglion (data not shown). $\gamma$-Synuclein was also closely approximated to the efferent synaptic marker synaptophysin with a nearly overlapping staining pattern (Fig. 3F, yellow). Co-labeling with choline acetyltransferase (ChAT) with the three synucleins also shows nearly identical approximation of the stains at the outer hair cell synaptic base (data not shown). Interestingly, both $\alpha$ - and $\gamma$ synuclein labeled the efferent tunnel crossing fibers as well as the efferent terminals, while the $\beta$-synuclein label appeared to be restricted to just the terminals. In

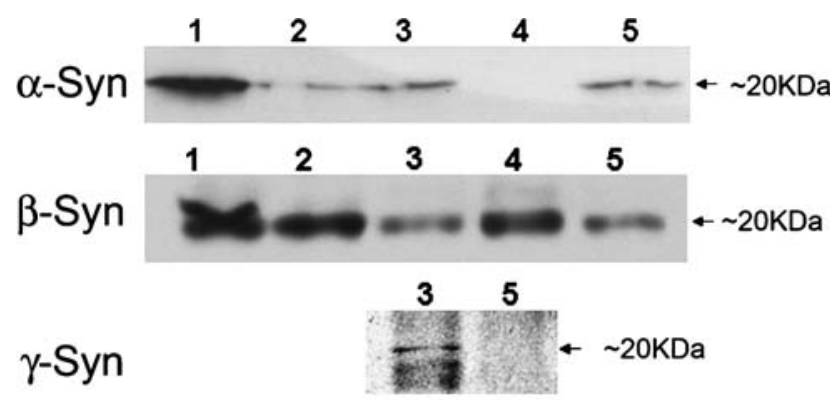

1-Mice spinal cord; 2-Rat cochlea; 3-Mouse-wild type cochlea; 4- $\alpha$-synuclein knockout cochlea; 5 ; $\gamma$-synuclein knockout cochlea.

FIG. 2. Western blot of synucleins in the cochlea. Western blot analysis of several tissue types was used to assess for the presence of the three synuclein proteins $(\alpha, \beta, \gamma)$, as demonstrated by the band at $\sim 20 \mathrm{kD}$ (arrow). In lane 1, mice spinal cord, served as a positive control for $\alpha$ and $\beta$-synuclein. $\alpha$ and $\beta$-Synuclein were both identified in rat coch lear tissue (lane 2). All three synucleins are identified in mouse cochlear tissue (lane 3). In the $\alpha$-synuclein knockout mouse (lane 4), the cochlear tissue appropriate detects $\beta$ - but not $\alpha$-synuclein. Similarly, in the $\gamma$ synuclein knockout mouse (lane 5), as expected, the cochlear tissues detect $\alpha$ - and $\beta$ - but not $\gamma$-synuclein. These later studies demonstrate the specificity of the antibodies used for subsequent studies as well as the correct phenotype of the null-mutant mice. 

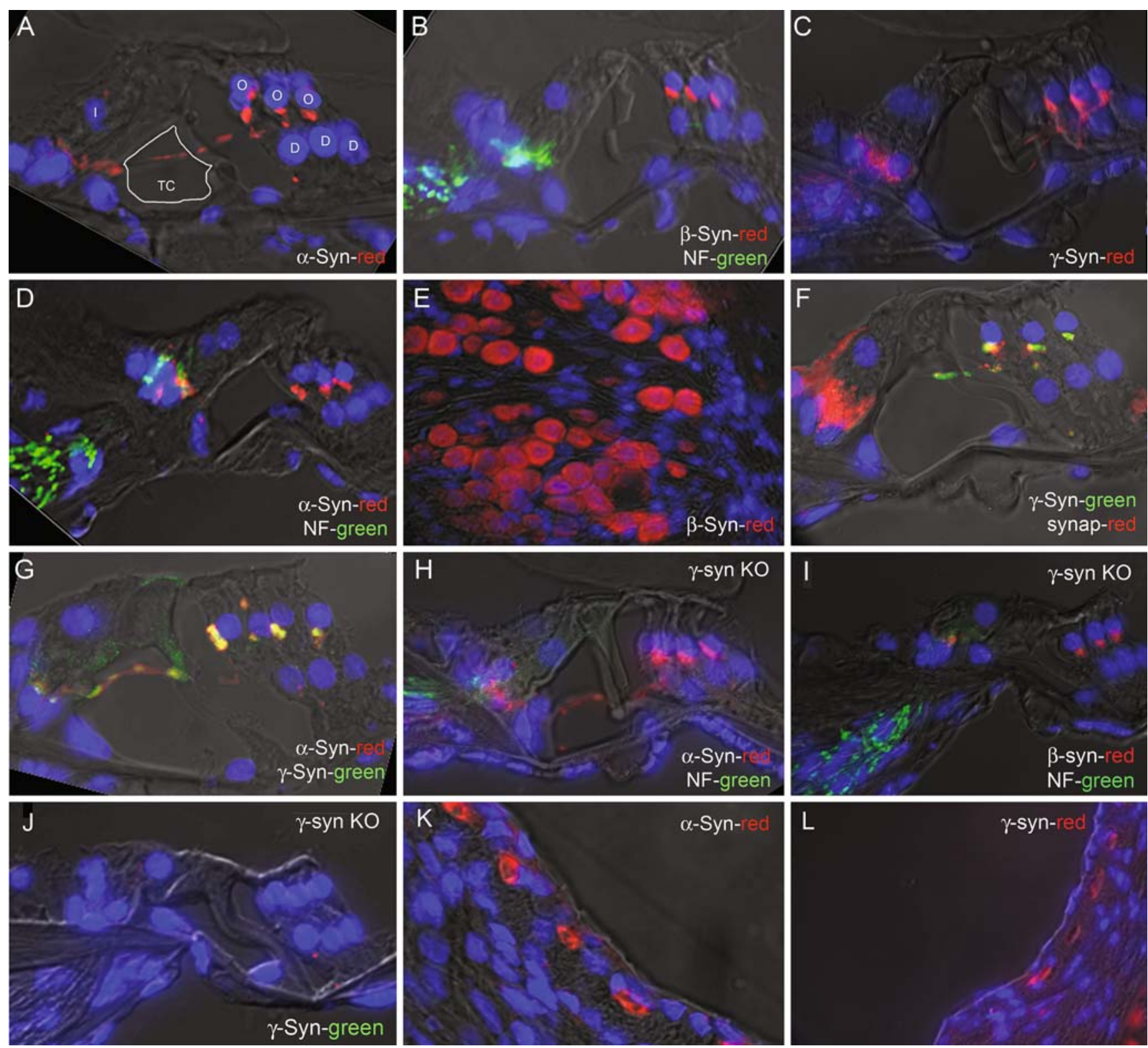

FIG. 3. Immunofluorescence of synucleins within the mouse cochlea. Single or double-label immunofluorescence localizes the three synucleins within the cochlea, predominantly to the efferent neuronal system and outer hair cell synaptic region. Outlines of relevant cochlear structures are provided in A ( $I$ Inner hair cell, $O$ outer hair cell, $D$ Deiters cell, $T C$ tunnel of Corti), while the orientation of all organ of Corti $(\mathbf{A}-\mathbf{D}, \mathbf{F}-\mathbf{I})$ are similar. $\alpha$-Synuclein localizes predominantly in the base of the outer hair cells and the efferent tunnel-crossing fibers (A, D, $\mathbf{G}, \mathbf{H})$ and more weakly in the base of the inner hair cell in the region of the inner spiral bundle, as well as within the stria vascularis $(\mathbf{K})$. In contrast, $\beta$-synuclein was most prominently localized to the synapse at the base of the outer hair cells (B, I), spiral ganglion (E), and more weakly within the stria vascularis (I). Antibodies against $\gamma$-synuclein most strongly label the efferent synapse at the base of the outer hair

the $\gamma$-synuclein knockout mouse (Fig. $3 \mathrm{~h}-\mathrm{j}$ ), there is no apparent change in the labeling pattern of $\alpha$-synuclein $(3 \mathrm{H}), \beta$-synuclein (3I) and an appropriate lack of $\gamma$ synuclein signal (3J), again demonstrating antibody specificity and the correct null-mutant phenotype. cells with some labeling of the efferent tunnel-crossing fibers $(\mathbf{C})$, and co-localizes with $\alpha$-synuclein at the efferent synapse near the base of the outer hair cell $(\mathbf{G})$, but shows no staining within the stria vascularis nor spiral ganglion (not shown). $\boldsymbol{\gamma}$-Synuclein is also closely approximated to the efferent synaptic marker synaptophysin with a nearly overlapping staining pattern (F). In the $\gamma$-synuclein null-mutant mouse $(\mathbf{H}-\mathbf{J})$, there is no apparent change in the labeling pattern of $\alpha$ synuclein $(\mathbf{H})$ or $\beta$-synuclein (I), while $\gamma$-synuclein appropriately shows no labeling in this mouse tissue (J). Together, these studies localize the three synucleins predominantly to the efferent neuronal system within the rodent organ of Corti with localization at the efferent outer hair cell synapse, with some additional localization within the efferent tunnelcrossing fibers ( $\alpha$ - and $\gamma$-synuclein), spiral ganglion ( $\beta$-synuclein), and stria vascularis ( $\alpha$ - > $\beta$-synuclein).

Together, these studies localize the three synucleins predominantly to the efferent neuronal system within the rodent cochlea with localization at the efferent outer hair cell synapse, with some additional localization within the efferent tunnel-crossing fibers $(\alpha-$ and $\gamma$ - 
synuclein), spiral ganglion ( $\beta$-synuclein), and stria vascularis $(\alpha->\beta$-synuclein $)$.

Developmental studies were next undertaken to determine the onset of synuclein production (Fig. 4).
These studies demonstrate that $\gamma$-synuclein has the earliest onset at E19, first detectable in the region below the outer hair cells (arrow, E19, $\gamma$-synuclein) and inner spiral bundle (asterisk, E19, $\gamma$-synuclein).

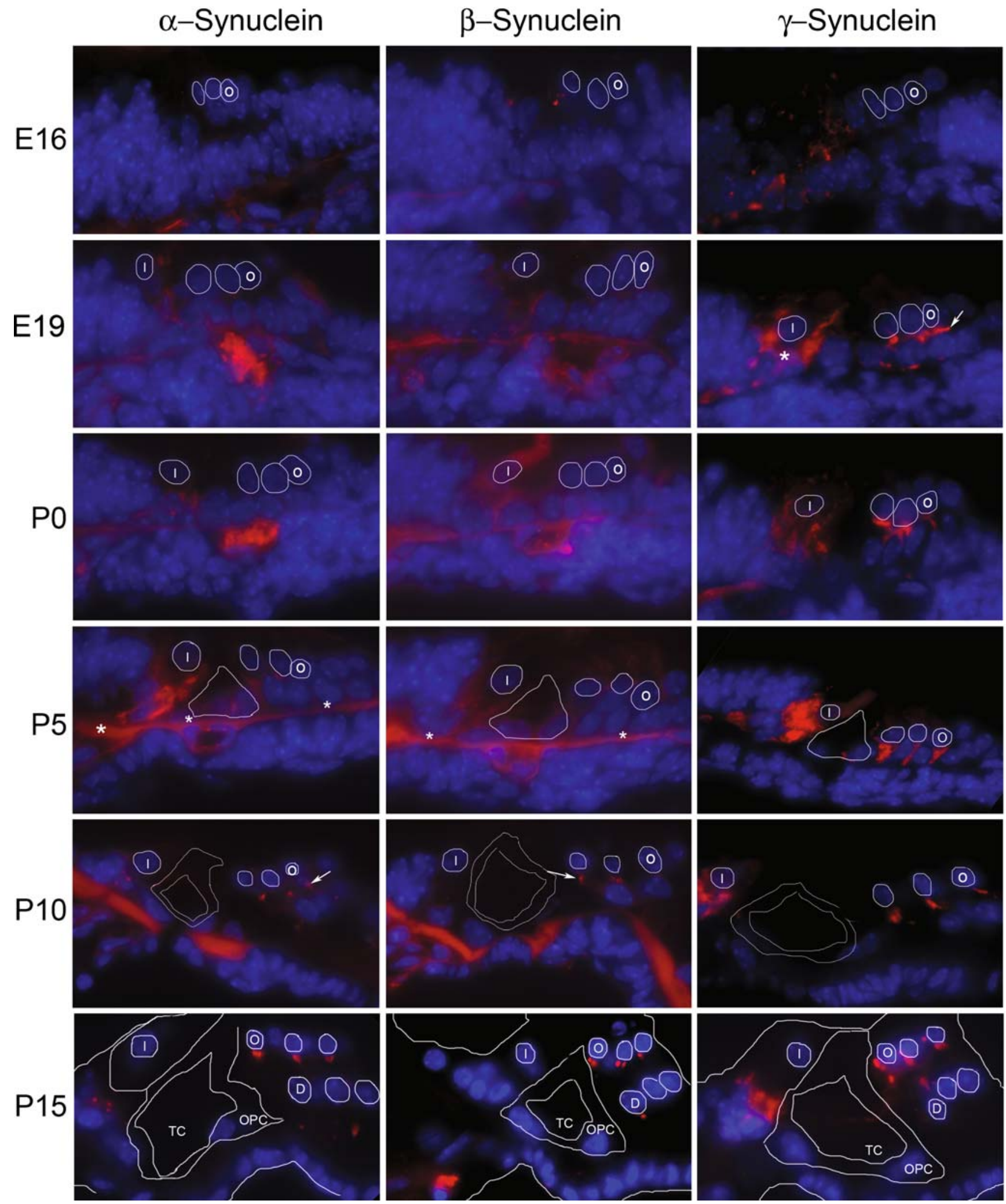


In contrast, immunofluorescent labeling of $\alpha$ - or $\beta$ synuclein cannot be clearly seen until P10 (arrows, P10, $\alpha$ - and $\beta$-synuclein) below the outer hair cells. Labeling in the region of the developing inner spiral bundle by $\gamma$-synuclein can be seen initially at E19, becomes more localized through P15, and persists through P21 (compare to Fig. 3c and 3f). Interestingly, for both $\alpha$ - or $\beta$-synuclein, linear labeling along the basilar membrane was consistently seen between E19 and P10 (asterisks, P5, $\alpha$ - and $\beta$-synuclein), but disappearing by $\mathrm{P} 15$. Whether this represents transient expression of synuclein during this time period or cross-reactivity with another protein is unclear, though blocking endogenous mouse IgG with a goat anti-mouse $\operatorname{IgG}(\mathrm{H}+\mathrm{L})$ antibody (Zymed) failed to eliminate the signal.

\section{$\gamma$-Synuclein null-mutant mice}

In an effort to understand synuclein function within the inner ear, a $\gamma$-synuclein knockout mouse was studied. The correct phenotype of the mouse was verified by genotyping and RT-PCR (Fig. 1), Western blot analysis (Fig. 2), and immunofluorescence (Fig. 3). Prior work has shown no obvious phenotypic changes in the $\gamma$-synuclein null mouse (Ninkina et al. 2003). As shown by Figure 5, we could not identify any morphologic changes in the organ of Corti in the knockout compared to the wild-type mouse; organ of Corti histology, outer and inner hair cell numbers, and spiral ganglion cell numbers were normal and identical in each zygosity (only wild-type and knockouts are shown in Figure 5). Furthermore, though $\alpha$ synuclein and, to a lesser extent, $\beta$-synuclein are found within the stria vascularis (Fig. 3), no abnormality was seen in this region either in the nullmutant mouse (data not shown).

FIG. 4. Developmental expression of synucleins in the cochlea. Synuclein expression was assessed at E16, E19, P1, P5, P10, and P15 to determine onset of $\alpha$-synuclein (left column), $\beta$-synuclein (middle column), and $\gamma$-synuclein (right column). These studies demonstrate that $\gamma$-synuclein has the earliest onset, first detectable in the region below the outer hair cells at approximately E19 (arrow, E19, $\gamma$-synuclein). In contrast, immunofluorescent labeling of $\alpha$ - or $\beta$-synuclein cannot be clearly seen until P10 (arrows, P10, $\alpha$ - and $\beta$-synuclein). Diffuse $\gamma$ synuclein label can also be seen below the base of the developing inner hair cell at the earliest time point studied (E16), becoming more localized through P15, but disappearing by P21 (compare to Fig. 3c and $\mathrm{f}$ ), an area corresponding to the developing inner spiral bundle. A linear labeling of unclear significance along the basilar membrane was consistently seen between E19 and P10 in the $\alpha$ - and $\beta$-synuclein sections, but disappearing by P15 for both (asterisks, P5). To assist with orientation, relevant structures are outlined that are identifiable for all three synucleins; these include developing inner hair cell nuclei ("I", P0 onward), tunnel of Corti ("TC", P5-P15), outer pillar cell ("OPC", P15 only), outer hair cell nuclei ("O", E16 onward) and Deiters' cell nucleus ("D", P15 only). The orientation for all figures is the same.

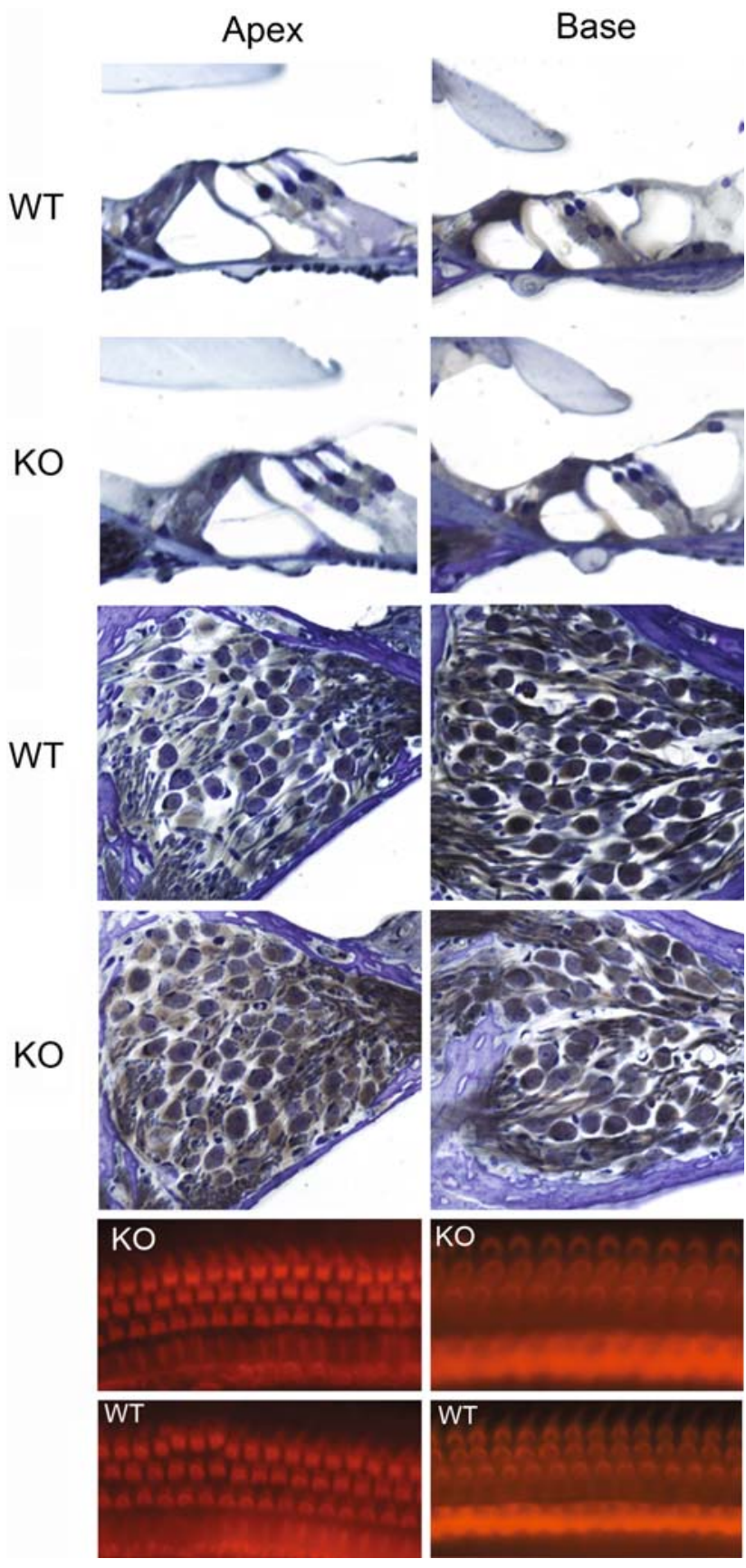

FIG. 5. Null-mutant $\gamma$-synuclein histology and surface preparation. Light microscopy (top eight panels) and surface preparation (bottom four panels) from the $\gamma$-synuclein null mutant mouse and wild-type littermate. Organ of Corti histology (upper four panels) and spiral ganglion region (middle four panels) from both the apex and base of the cochlea are included for comparison. These studies demonstrate no significant histological differences between the wild-type (WT) and knockout (KO) mice; each show normal light microscopy morphology and spiral ganglion cells. Similarly, surface preparations from these same mice (WT and KO) from both the apex and base demonstrate normal inner and outer hair cells.

$\mathrm{ABR}$ and DPOAE testing were performed to evaluate the hearing and gross outer hair cell function in the knockout mouse. As shown (Fig. 6), the knockout mice $(n=10)$ have similar hearing thresholds and outer hair 
cell function at the age tested (P21) compared to wildtype mice $(n=6)$. For DPOAEs, only the 60 -db tracings are shown; there also was no difference noted between WT and KO mice at $65 \mathrm{db}$ (data not shown). Based upon these studies, it can be concluded that $\gamma$ synuclein function alone is not required for the onset and maintenance of normal hearing and outer hair cell function at least through P21.

\section{DISCUSSION}

Despite widespread localization of synucleins throughout the CNS, their exact function remains undetermined. However, with their identification in Lewy bodies, the pathological hallmark of disorder of Parkinson's disease (Spillantini et al. 1997) and their role in other neurodegenerative disorders, interest in the underlying physiology of synucleins remains high. The identification of synucleins within the auditory neuronal system also now raises some interesting questions regarding their possible role in neurodegenerative processes within the inner ear.

This initial work focuses on localization of the three synucleins within the mammalian organ of Corti, and our results correlate with prior localization studies of synucleins within the CNS. Prior work by other groups have shown strong labeling of the synucleins throughout the CNS, with both $\alpha$ - and $\beta$-synuclein migrating from the cell body to the presynaptic nerve terminals at approximately $17-20$ weeks and $\beta$ synuclein ultimately localizing to somatic cholinergic neurons. In contrast, $\gamma$ synuclein is localized primarily to the peripheral nervous system in both cholinergic and catecholaminergic neurons (Jakes et al. 1994; Lavedan et al. 1998a; Lavedan et al. 1998b; Li et al. 2002; Ueda et al. 1993). Here, we identified all three synucleins, $\alpha-, \beta-$, and $\gamma$-, within the rodent cochlea using multiple modalities (RT-PCR, Western blot, and immunofluorescence). The three synucleins all localize predominantly to the efferent synapse at the base of the outer hair cell, colocalizing both with synaptophysin (Fig. 3) and ChAT (data not shown). Furthermore, there is additional weaker labeling of the base of the inner hair cell in the region of the inner spiral bundle ( $\alpha$ - and $\gamma$-synuclein) and efferent tunnel-crossing fibers ( $\alpha$ - and $\gamma$ - synucleins). Thus, the previously described cholinergic localization of synucleins within the CNS is recapitulated in the peripheral organ of Corti. The significance of the additional labeling seen in the spiral ganglion cells ( $\beta$-synuclein), and stria vascularis $(\alpha-$ and $\beta$ synuclein; Fig. 3) is unclear.

Interestingly, most studies show that both $\alpha$ - and $\beta$ synuclein have similar expression patterns. In this study, $\beta$-synuclein appears to differ from both $\alpha$ - and $\gamma$ synuclein in at least two regards: $\beta$-synuclein was the

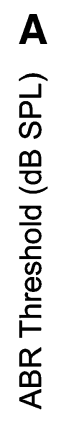
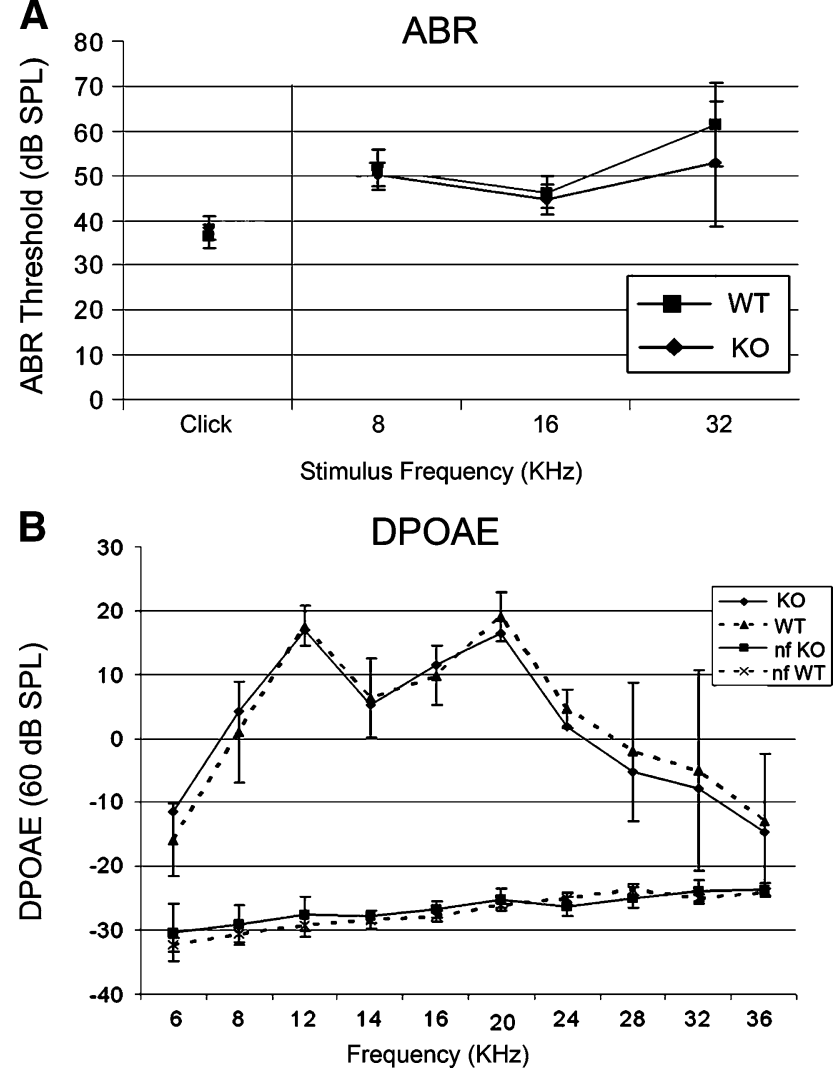

FIG. 6. Auditory physiology in the $\gamma$-synuclein null mutant mouse. Auditory brainstem response ( $A B R, A)$ threshold testing as well as distortion product otoacoustic emissions (DPOAE, B) testing was used to test hearing in the $\gamma$-synuclein knockout mouse compared to the wild-type littermate. ABR testing of both broadband clicks and toneburst stimuli (A) demonstrates no significant differences in hearing between the wild-type (WT $n=6)$ and knockout (KO) mice $(n=10)$. Similarly, DPOAE testing (B) demonstrated no significant difference between the wild-type (WT) and knockout (KO) mice. The reference noise floor ( $\mathrm{nf} \mathrm{KO}$, nf WT) of each DPOAE tracing is included.

only one to label the spiral ganglion neurons (Fig. 3e), and in contrast to both $\alpha$-and $\gamma$-synuclein which labeled both efferent tunnel crossing fibers (medial olivocochlear fibers) and the efferent terminals, $\beta$-synuclein only labeled the efferent terminals. $\beta$-synuclein also showed the highest levels of mRNA by qPCR in the cochlea (Fig. 1). Whether this might represent different functions between the synucleins remains unclear, though it would appear from these results that $\beta$ synuclein has a distinct role to play within the cochlea.

The developmental labeling pattern identified is somewhat interesting. Labeling below the developing outer hair cells was seen as early as E19 for $\gamma$-synuclein, while $\alpha$ - and $\beta$-synuclein did not label their respective regions until $\sim$ P10, implying two different functions for these isoforms. Studies by Fritzsch (1996) and Karis et al. (2001) show that the first efferent fibers can be traced towards various sensory epithelia of the inner ear by E13 and penetrate the sensory neuroepithelia by E14E15 with individual efferent axons branching below 
OHCs at this time. While some studies show that the first observable efferent synapse on hair cells do not occur until approximately P0, additional studies (Bruce et al. 2000; Emmerling et al. 1990) suggest that the efferent axons and their growth cones may reach hair cells much earlier than this, before the first synapses can be identified (Simmons 2002). Thus the $\gamma$-synuclein labeling we identified below the $\mathrm{OHC}$ at E19 and beyond might be consistent with this notion, though what the precise role of $\gamma$-synuclein would be is unclear at present. In contrast, $\alpha$ - and $\beta$-synuclein, not showing up until P10, may play a more traditional role in efferent synaptic function.

Within the CNS, the function of synucleins in the cochlea and their relationship to hearing remains unclear. Prior studies have shown that synucleins may play a role in synaptic function. In mature cultured primary neurons, synucleins co-localize almost exclusively with synaptophysin in the presynaptic terminals and appear earlier than synaptophysin, but later than the vesicleassociated protein synapsin I during CNS development (Hsu et al. 1998; Jensen et al. 1999; Murphy et al. 2000; Withers et al. 1997). Additional recent studies have shown that $\alpha$-synuclein may be required for the genesis and/or maintenance of resting pools of presynaptic vesicles (Cabin et al. 2002; Gureviciene et al. 2007; Murphy et al. 2000). Recent work has also shown that $\alpha$ synuclein can potentiate $\mathrm{Ca}^{2+}$ influx through voltagedependent $\mathrm{Ca}^{2+}$ channels (Adamczyk and Strosznajder 2006; Ueda et al. 1997). The identification of all three synucleins at the base of the outer hair cell at the efferent synapse, co-localizing with both synaptophysin and ChAT would therefore support a similar role for synucleins in efferent neuronal transmission in the auditory system. Perhaps through regulation of the size of the resting pool of presynaptic vesicles, as has been demonstrated in cultured neurons (Cabin et al. 2002; Murphy et al. 2000), synucleins may play a role in longterm, sustained release of acetylcholine in the efferent auditory system.

Prior work has shown that $\alpha$-synucleins can exist in various splice forms and that variations in these splice forms may be part of the underlying pathophysiology in such neurodegenerative disorders as Parkinson's disease.(Beyer et al. 2008; Fuchs et al. 2008; Muller et al. 2005) It is thus intriguing to speculate that a similar phenomenon may be occurring in degenerative auditory processes. While synuclein isoforms were not specifically sought in this work, it would certainly be worthy of further investigation.

As this study demonstrates, $\gamma$-synuclein alone is not required for the onset or maintenance of normal hearing and outer hair cell function through P21, as demonstrated by the knockout mouse. Our studies show no phenotypic or histological differences in the inner ears of the wild-type vs. the knockout mice, while ABR thresholds and otoacoustic emissions also are unchanged in the knockout mouse through P21. This follows prior studies on this same mouse, in which no difference was observed in the number of neurons between wild-type and null mutant animals in several brain stem motor nuclei, in lumbar dorsal root ganglia, and in the trigeminal ganglion (Ninkina et al. 2003). Lack of clear morphological and phenotypic differences were similarly observed in the CNS of $\alpha$-synuclein null mutant mouse (Abeliovich et al. 2000; Cabin et al. 2002). Whether the $\alpha$-synuclein null mutant mouse has any auditory defect is still unknown, though it is currently under study in our lab. Furthermore, in contrast to the rodent CNS (Li et al. 2002), our data indicates that $\beta$-synuclein is the predominant mRNA species in the cochlea (Fig. 1) and that perhaps a $\beta$ synuclein null-mutant mouse would show auditory defects; unfortunately a $\beta$-synuclein knockout mouse is not currently available to us for study. Based upon prior findings, it has been postulated that the close homologous protein structure as well as overlapping expression patterns of all three synucleins allow the other synucleins to compensate for loss of one isoform as seen in the null mutant mice studied to date(Ninkina et al. 2003). This could certainly account for a lack of any auditory deficits within the cochlea in the $\gamma$-synuclein knockout mice studied here. Arguing against this proposition, however, is that prior studies have failed to demonstrate altered expression levels of the other synucleins in either the $\alpha$ - and $\gamma$-synuclein null mutants (Abeliovich et al. 2000; Ninkina et al. 2003). However, studies on double mutant mice lacking both $\alpha$ - and $\beta$ synuclein have demonstrated that, while synucleins are not essential components of synaptic transmission, they may contribute to the long-term regulation or maintenance of presynaptic function through their effects on transmitter release(Chandra et al. 2004).

Despite not finding an obvious deficit within the inner ears of the $\gamma$-synuclein null mutant mice, the organ of Corti nonetheless could be an important model for testing synuclein function, a protein known to be involved in other neurodegenerative disorders. There are a variety of neurodegenerative processes that can affect the inner ear, most notably presbycusis or agerelated hearing loss. Recent reports have linked $\alpha$ synuclein to voltage-gated calcium flux, known to play a role in outer hair cell efferent signaling (Adamczyk and Strosznajder 2006). Furthermore, Cabin et al. (2002) have shown that, while there were no obvious phenotypic changes in an $\alpha$-synuclein knockout mouse, prolonged trains of repetitive stimulation in hippocampal slice preparations depleted both docked and reserve pools of neurotransmitter vesicles, while replenishment of these vesicles were slower in the mutant synapses. Thus, the localization of synucleins predominantly to the efferent neuronal system within 
the inner ear raises the intriguing possibility that synuclein dysfunction could play a role in susceptibility to noise-induced hearing loss or presbycusis, two disorders that have been linked, in part, to the efferent neuronal system (Maison and Liberman 2000; Zhu et al. 2007). The finding of $\alpha$ - and $\beta$-synuclein within the stria vascularis, also a well-known site of age-related hearing loss changes (Spicer and Schulte 2005), further supports this notion. Thus, it is possible that age-related hearing loss studies beyond P21, or studies with auditory perturbation (e.g., loud noise exposure) could reveal deficits not seen in our initial studies. Such studies are currently ongoing in our lab in an attempt to better define the role of synucleins within the inner ear.

\section{ACKNOWLEDGEMENTS}

We would like to acknowledge the support of Hearing Research Inc, Dr. Bernard Kramer, and NIH NIDCD K08 DC00189 (Lustig), as well as The Wellcome Trust (Buckman).

\section{REFERENCES}

Abeliovich A, Schmitz Y, Farinas I, Choi-Lundberg D, Ho WH, Castillo Pe, Shinsky N, Verdugo JM, Armanini M, Ryan A, et al. Mice lacking alpha-synuclein display functional deficits in the nigrostriatal dopamine system. Neuron. 25:239-252, 2000.

Adamczik A, Strosznajder JB. Alpha-synuclein potentiates $\mathrm{Ca}^{2+}$ influx through voltage-dependent $\mathrm{Ca}^{2+}$ channels. Neuroreport. 17: 1883-1886, 2006.

Akil O, Chang J, Hiel H, Kong JH, Yi E, Glowatzki E, Lustig LR. Progressive deafness and altered cochlear innervation in knockout mice lacking prosaposin. J. Neurosci. 26:13076-13088, 2006.

Berglund AM, Ryugo DK. Neurofilament antibodies and spiral ganglion neurons of the mammalian cochlea. J. Comp. Neurol. 306:393-408, 1991.

Beyer K, Domingo-Sabat M, Humbert J, Carrato C, Ferrer I, Ariza A. Differential expression of alpha-synuclein, parkin, and synphilin1 isoforms in Lewy body disease. Neurogenetics 9:163-172, 2008.

Bruce LL, Christensen MA, WARR WB. Postnatal development of efferent synapses in the rat cochlea. J. Comp. Neurol. 423:532-548, 2000.

Buchman VL, Adu J, Pinon LG, Ninkina NN, Davies AM. Persyn, a member of the synuclein family, influences neurofilament network integrity. Nat. Neurosci. 1:101-103, 1998.

Cabin De, Shimazu K, Murphy D, Cole NB, Gottschalk W, Mcilwain KL, Orrison B, Chen A, Ellis CE, Paylor R, et al. Synaptic vesicle depletion correlates with attenuated synaptic responses to prolonged repetitive stimulation in mice lacking alpha-synuclein. J. Neurosci. 22:8797-8807, 2002.

Chandra S, Fornai F, Kwon HB, Yazdani U, Atasoy D, Liu X, Hammer RE, Battaglia G, German DC, Castillo PE, et al. Doubleknockout mice for alpha- and beta-synucleins: effect on synaptic functions. Proc. Natl. Acad. Sci. U S A 101:14966-14971, 2004.

Emmerling MR, Sobkowicz HM, LeVenick CV, Scott GL, SLAPNick SM, Rose JE. Biochemical and morphological differentiation of acetylcholinesterase-positive efferent fibers in the mouse cochlea. J. Electron. Microsc. Tech. 15:123-143, 1990.

EyBaLIN M. Neurotransmitters and neuromodulators of the mammalian cochlea. Physiol. Rev. 73:309-373, 1993.
Fritzsch B. Development of the labyrinthine efferent system. Ann. N. Y. Acad. Sci. 781:21-33, 1996.

Fuchs J, Tichopad A, Golub Y, Munz M, Schweitzer KJ, Wolf B, Berg D, Mueller JC, Gasser T. Genetic variability in the SNCA gene influences alpha-synuclein levels in the blood and brain. Faseb. J. 22:1327-1334, 2008.

George JM. The synucleins. Genome. Biol. 3:3001-3006, 2002, REVIEWS3002.

Gureviciene I, Gurevicius K, Tanila H. Role of alpha-synuclein in synaptic glutamate release. Neurobiol. Dis. 28:83-89, 2007.

Hsu LJ, Mallory M, Xia Y, Veinbergs I, Hashimoto M, Yoshimoto M, Thal LJ, Sattoh T, Masliah E. Expression pattern of synucleins (non-Abeta component of Alzheimer's disease amyloid precursor protein/alpha-synuclein) during murine brain development. J. Neurochem. 71:338-344, 1998.

Jakes R, Spillantini MG, Goedert M. Identification of two distinct synucleins from human brain. FEBS Lett. 345:27-32, 1994.

Jenco JM, Rawlingson A, Daniels B, Morris AJ. Regulation of phospholipase D2: selective inhibition of mammalian phospholipase D isoenzymes by alpha- and beta-synucleins. Biochemistry 37:4901-4909, 1998.

Jensen PH, Li JY, Dahlstrom A, Dotti CG. Axonal transport of synucleins is mediated by all rate components. Eur. J. Neurosci. 11:3369-3376, 1999.

KAHLE PJ. Alpha-synucleinopathy models and human neuropathology: similarities and differences. Acta Neuropathol. 115:87-95, 2007.

Karis A, Pata I, van Doorninck JH, Grosveld F, de Zeeuw Ci, de Caprona D, Fritzsch B. Transcription factor GATA-3 alters pathway selection of olivocochlear neurons and affects morphogenesis of the ear. J. Comp. Neurol. 429:615-630, 2001.

Lavedan C, Buchholtz S, Auburger G, Albin RL, Athanassiadou A, Blancato J, Burguera JA, Ferrell RE, Kostic V, Leroy E, et al. Absence of mutation in the beta- and gamma-synuclein genes in familial autosomal dominant Parkinson's disease. DNA Res. 5:401-402, 1998a.

Lavedan C, Leroy E, Torres R, Dehejia A, Dutra A, Buchholtz S, Nussbaum RL, Polymeropoulos MH. Genomic organization and expression of the human beta-synuclein gene (SNCB). Genomics 54:173-175, 1998b.

Li JY, Henning Jensen P, Dahlstrom A. Differential localization of alpha-, beta- and gamma-synucleins in the rat CNS. Neuroscience 113:463-478, 2002.

Livak KJ, Schmittgen TD. Analysis of relative gene expression data using real-time quantitative PCR and the 2(-Delta Delta C(T)) method. Methods 25:402-408, 2001.

Maison SF, Liberman MC. Predicting vulnerability to acoustic injury with a noninvasive assay of olivocochlear reflex strength. J. Neurosci. 20:4701-4707, 2000.

Muller CM, de Vos RA, Maurage CA, Thal DR, Tolnay M, Braak H. Staging of sporadic Parkinson disease-related alpha-synuclein pathology: inter- and intra-rater reliability. J. Neuropathol. Exp. Neurol. 64:623-628, 2005.

Murphy DD, Rueter SM, Trojanowski JQ Lee VM. Synucleins are developmentally expressed, and alpha-synuclein regulates the size of the presynaptic vesicular pool in primary hippocampal neurons. J. Neurosci. 20:3214-3220, 2000.

Ninkina N, Papachroni K, Robertson DC, Schmidt O, Delaney L, O’Neill F, Court F, Rosenthal A, Fleetwood-Walker SM, Davies AM, ET AL. Neurons expressing the highest levels of gammasynuclein are unaffected by targeted inactivation of the gene. Mol. Cell. Biol. 23:8233-8245, 2003.

Puel JL. Chemical synaptic transmission in the cochlea. Prog. Neurobiol. 47:449-476, 1995.

Schimmang T, Tan J, Muller M, Zimmermann U, Rohbock K, Kopschall I, Limberger A, Minichiello L, KNipPer M. Lack of Bdnf and TrkB signalling in the postnatal cochlea leads to a spatial reshaping of innervation along the tonotopic axis and hearing loss. Development 130:4741-4750, 2003. 
Simmons DD. Development of the inner ear efferent system across vertebrate species. J. Neurobiol. 53:228-250, 2002.

SpICER SS, Schulte BA. Pathologic changes of presbycusis begin in secondary processes and spread to primary processes of strial marginal cells. Hear Res. 205:225-240, 2005.

Spillantini MG, Schmidt ML, Lee VM, Trojanowski JQ Jakes R, Goedert M. Alpha-synuclein in Lewy bodies. Nature 388:839-840, 1997.

STichel CC, Zhu XR, Bader V, Linnartz B, Schmidt S, Lubbert H. Monoand double-mutant mouse models of Parkinson's disease display severe mitochondrial damage. Hum. Mol. Genet. 16:3377-3393, 2007.

Tofaris GK, Spillantini MG. Physiological and pathological properties of alpha-synuclein. Cell Mol. Life Sci. 64:2194-2201, 2007.

Ueda K, Fukushima H, Masliah E, Xia Y, Inai A, Yoshimoto M, Otero DA, Kondo J, Ihara Y, SAITOH T. Molecular cloning of cDNA encoding an unrecognized component of amyloid in Alzheimer disease. Proc. Natl. Acad. Sci. U S A 90:11282-11286, 1993.

Ueda K, Shinohara S, Yagami T, Asakura K, Kawasaki K. Amyloid beta protein potentiates $\mathrm{Ca}^{2+}$ influx through L-type voltage-sensitive $\mathrm{Ca}^{2+}$ channels: a possible involvement of free radicals. J. Neurochem. 68:265-271, 1997.

Withers GS, George JM, Banker GA, Clayton DF. Delayed localization of synelfin (synuclein, NACP) to presynaptic terminals in cultured rat hippocampal neurons. Brain Res. Dev. Brain Res. 99:87-94, 1997.

Zhu X, Vasilyeva ON, Kim S, Jacobson M, Romney J, Waterman MS, Tuttle D, Frisina RD. Auditory efferent feedback system deficits precede age-related hearing loss: contralateral suppression of otoacoustic emissions in mice. J. Comp. Neurol. 503:593-604, 2007. 